\title{
Trabectome Patient Selection, Preparation, Technique, Management, and Outcomes
}

\author{
Hardik A Parikh, BSc, ${ }^{1,2}$ Pritha Roy, MD, ${ }^{3}$ Amar Dhaliwal, BSc, ${ }^{4}$ Kevin B Kaplowitz, $\mathrm{MD}^{5}$ and Nils A Loewen, $\mathrm{MD}, \mathrm{PhD}^{6}$ \\ 1. Medical Student, Rutgers State University of New Jersey, Newark, New Jersey, US; 2. Research Fellow, University of Pittsburgh School of Medicine, Pittsburgh, \\ Pennsylvania, US; 3. Clinical and Research Fellow, University of Pittsburgh School of Medicine, Pittsburgh, Pennsylvania, US; 4. Medical Student, Department of \\ Ophthalmology, University of Pittsburgh School of Medicine, Pittsburgh, Pennsylvania, US; 5. Assistant Professor of Ophthalmology, Department of Ophthalmology, \\ Stony Brook University School of Medicine, Stony Brook, NY, US; 6. Assistant Professor of Ophthalmology, Department of Ophthalmology, University of Pittsburgh School of \\ Medicine, Pittsburgh, Pennsylvania, us
}

\begin{abstract}
Ab interno trabeculectomy with the Trabectome ${ }^{\circledR}$ (NeoMedix, Tustin, CA, US) is a minimally invasive glaucoma surgery that has the capacity to significantly lower intraocular pressure (IOP) with a highly favorable safety profile compared with traditional glaucoma surgeries. Since the first results were reported in 2005 , mostly for primary open-angle glaucoma, the indications have since expanded to include secondary open- and closed-angle glaucoma. Exclusion criteria are active neovascular and uveitic glaucoma, and glaucoma due to increased episcleral pressure. On average, IOP is typically lowered by $30-40 \%$ into the midteens, while decreasing the number of medications by one. Postoperative IOP can be estimated using a calculator. Patients with a higher preoperative IOP have the largest IOP reduction. Transient hyphema is a common postoperative observation. In this article, we will review surgical technique, patient management, postoperative care, and outcomes.
\end{abstract}

\section{Keywords}

Trabectome, ab interno trabeculectomy, minimally invasive glaucoma surgery, glaucoma, plasma surgery, surgical technique

Disclosure: Hardik A Parikh, BSC, Pritha Roy, MD, Amar Dhaliwal, BSC, and Kevin B Kaplowitz, MD, have no relevant conflicts of interest to declare. Nils A Loewen, MD, PhD, has received honoraria from NeoMedix as a Trabectome wet lab trainer and lecturer. No funding was received in the publication of this article.

Open Access: This article is published under the Creative Commons Attribution Noncommercial License, which permits any noncommercial use, distribution, adaptation, and reproduction provided the original author(s) and source are given appropriate credit.

Received: August 10, 2015 Accepted: September 17, 2015 Citation: US Ophthalmic Review, 2015;8(2):103-7 DOI: http://doi.org/10.17925/USOR.2015.08.02.103 Correspondence: Nils Loewen, MD, PhD, 203 Lothrop St, Pittsburgh, PA 15213, US. E: Ioewenna@upmc.edu

Ab interno trabeculectomy with the Trabectome ${ }^{\circledR}$ (NeoMedix , Tustin, CA, US) is a minimally invasive glaucoma surgery (MIGS) modality that increases conventional outflow over 6 clock hours through a single incision. ${ }^{1}$ The tip of the Trabectome handpiece generates plasma that ionizes and ablates the trabecular meshwork (TM), a mechanism that is fundamentally different from cautery. The primary mode of action is enhancement of conventional outflow along the physiologic route and in this regard is similar to TM bypass microstents, ${ }^{2,3}$ which produce a more segmentally limited outflow. ${ }^{4}$ The growing prevalence of glaucoma, 5,6 along with increasing longevity and the desire to maintain physical, social, and occupational fitness, underscores the need for effective and well-tolerated glaucoma surgeries. Trabeculectomies and tube shunts, while effective, are associated with serious risks that amounted to $74 \%$ of trabeculectomies and $27 \%$ of tubes needing manipulation. Thirty-nine percent of trabeculectomies had early and $38 \%$ had late vision-threatening complications (total of $77 \%$ ) compared with tube shunts with $22 \%$ early and $36 \%$ (total of $58 \%$ ) experiencing vision-threatening complications during 5 years follow up. ${ }^{7}$ Other patient populations and surgeons have reported more favorable results, but these studies were not nonrandomized controlled. 8,9 As MIGS are standardized with predictable surgeon factors, they can be well combined with cataract surgery and allow implantation of advanced intraocular lenses.

The Trabectome (see Figure 1) was granted US Food and Drug Administration (FDA) approval in the US in April 2004 for use in adult as well as pediatric glaucoma as the only device specifically approved for this population. ${ }^{10}$ The tip design was updated in 2012 with a more beveled sleeve to allow easier entry and larger irrigation ports for improved chamber stability. Use of viscoelastic prior to Trabectome surgery was discouraged and removed from the package. The Trabectome has gained widespread international adoption in the decade following its introduction. ${ }^{11,12}$ The current commercial device was developed in conjunction with the NeoMedix Corporation, who continues to distribute and market the device platform.

\section{Trabectome Patient Selection}

The original studies of Trabectome surgery included an indication of glaucoma with an angle open to at least $20^{\circ}$ and excluded narrow angles and neovascular glaucoma. ${ }^{13}$ Subsequent studies have shown that Trabectome may also be effective in eyes with a narrow to very narrow angle. ${ }^{11}$ We 
Figure 1: Trabectome Handpiece (A, Left), Irrigation and Aspiration Console (B), and High-frequency Generator (C)

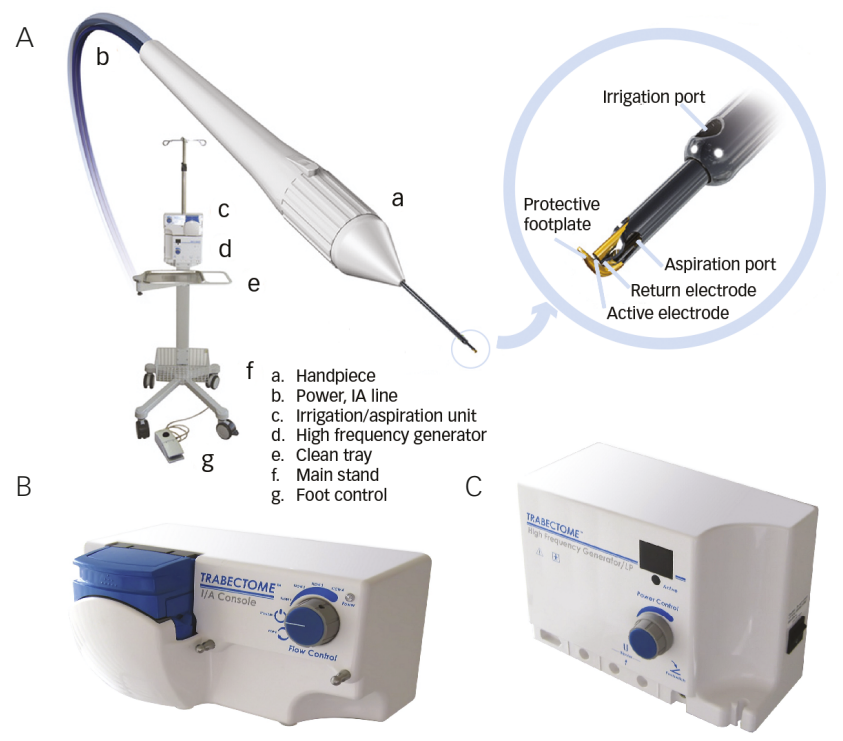

Figure 2: One-year IOPs (y-axis) Drop Toward $16 \mathrm{mmHg}$ Regardless of Preoperative IOP (X-axis) ${ }^{11}$
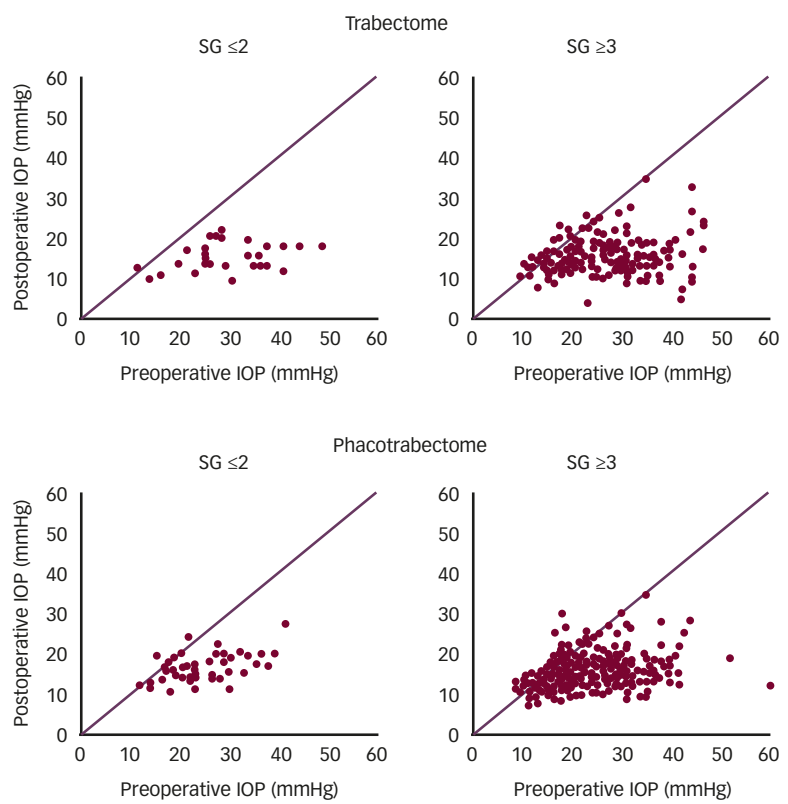

Results for narrow angles (Shaffer Grade $[S G] \leq 2$ ) are similar to deep angles $(S G \geq 3)$. IOP= intraocular pressure.

have operated on a wide range of secondary glaucomas, including following trauma, scleral buckle, uveitis, ${ }^{14}$ and failed trabeculectomies ${ }^{12}$ with successful lowering of intraocular pressure (IOP). The only remaining contraindications include active neovascular, active uveitic, or glaucoma due to increased episcleral pressure, since none of these would be thought to respond sufficiently to TM ablation.

Since successful Trabectome surgery frequently lowers the IOP into the midteens with relatively little influence of baseline IOP as evidenced by the single IOP dot plots ${ }^{11,12}$ (see results section, Figure 2), there does not appear to be an ideal baseline as patients with a higher preoperative IOP have a larger reduction that can be estimated with a formula as described below. The theoretical limit to postoperative IOP is the episcleral venous pressure. The severity of glaucoma does not appear to negatively influence IOP outcome. ${ }^{12}$ On the contrary, glaucomas with a more diseased TM and a higher relative glaucoma disease index show a larger IOP drop (unpublished data). Rather than focusing on the absolute decrease in IOP, some surgeons use a reduction of medication dependence as an indication, while others use Trabectome to treat ocular hypertension.

\section{Trabectome as the Initial Glaucoma Surgery}

No randomized controlled trial has formally compared Trabectome surgery to trabeculectomy or tube shunt surgery. Nonmatched, retrospective comparisons have reported conflicting results. Francis et al., found that while the average IOP was lower after trabeculectomy at 1 year, the success rate was $95 \%$ in patients who underwent phacotrabectome surgery compared with $83 \%$ in the phacotrabeculectomy group using the same success criteria (IOP $<21 \mathrm{mmHg}$ and no secondary surgery and IOP reduction $\geq 20 \%$ ). ${ }^{15}$ Mosaed et al. found a range of $55 \%$ to $95 \%$ after Trabectome only compared with $86.5 \%$ after trabeculectomy only. ${ }^{16}$ Jea et al. ${ }^{17}$ found a considerably lower success rate for Trabectome surgery.

The main reasons for selecting Trabectome as a first choice over trabeculectomies and tube shunt surgeries are that the procedure is faster than penetrating surgery, has a more routine postoperative course with fast visual recovery, and significantly fewer complications. The complication profile is not unlike that of cataract surgery with the exception of postoperative hyphema. Despite the high frequency of hyphema, ${ }^{17}$ a total of only three cases have been reported where intervention was necessary. ${ }^{18,19}$ Anticoagulants such as aspirin, coumadin, or antiplatelet agents can be safely continued. Since the surgery does not involve the conjunctiva, there should be no significant effect in cases when penetrating surgery is needed after unsuccessful Trabectome. Indeed, one of the first Trabectome investigations analyzed trabeculectomy after a failed Trabectome and found no difference in IOP or complications compared with a group undergoing primary trabeculectomies. ${ }^{20}$ Trabectome surgery has also demonstrated success without same session cataract surgery, after failed trabeculectomy, ${ }^{12}$ after tube shunts, ${ }^{21}$ canaloplasty, laser trabeculoplasty, ${ }^{22}$ and other complex glaucomas that include trauma, scleral buckles, ${ }^{14}$ and strabismus surgery.

\section{Patient Considerations}

Trabectome is ideal for active patients who need predictable visual recovery, do not want to have the longer-term risks associated with a bleb from trabeculectomy, or who want to wear contact lenses. Although predicting individual outcomes can only go so far, a new Trabectome surgery calculator, ${ }^{23}$ based on data of more than 600 patients in a matched comparison, can help to guide the surgeon in establishing preoperative expectations. The approximate IOP reduction can be predicted with the formula $-13.54+0.73 *($ phaco; yes:1, no:0)+0.73*(baseline 1OP)+0.59*(secondary open-angle glaucoma [SOAG]; yes:1, no:0)+0.03*(age)+0.09*(medications). This can aid in determining if the likely final IOP is within the patient's target range or if eye drops will be needed.

Cataracts can be removed in the same session. Since only a standardized clear corneal incision is used with a predictable surgeon factor, astigmatic 
Figure 3: Cross-section of Proper Engagement of the TM with Trabectome Handpiece and TM Threaded Between the Electrode Tips and Footplate (Left). Gonioscopic Visualization of the TM (Right)

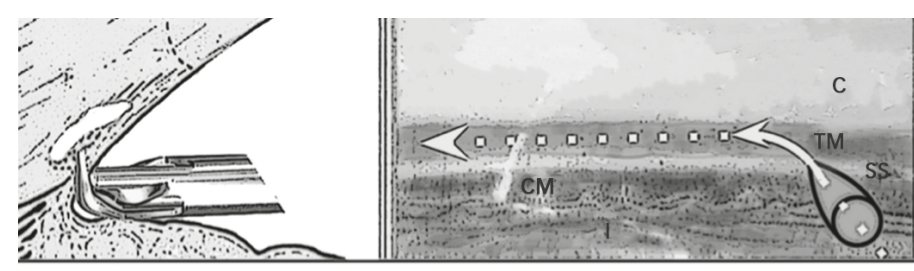

$C=$ cornea; $C B=$ ciliary body; $I=$ iris; $S S=$ scleral spur; $T M=$ trabecular meshwork.

Figure 4: Gaping the Main Incision and Inducing Hypotony Causes Blood to Reflux into SC

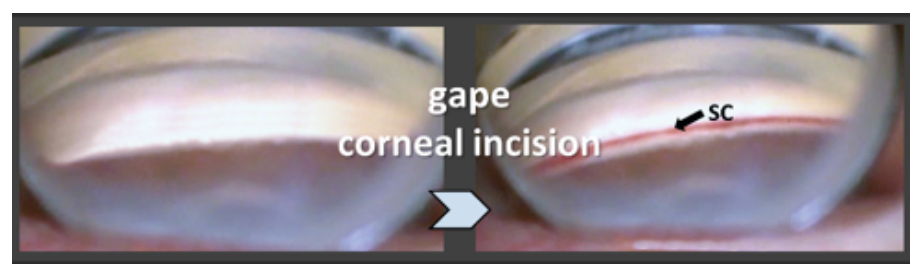

This procedure to identify the trabecular meshwork as the proper target and avoids confusion with the ciliary body band. $\mathrm{SC}=$ Schlemm's canal.

lenses can be safely implanted. Other situations that may see greater benefits from choosing Trabectome are patients in whom frequent postoperative visits and potential manipulations (i.e. reforming a flat anterior chamber) would present a great challenge, and patients, such as high myopes, who are at an increased risk for complications of hypotony including hypotony maculopathy, choroidals, and a flat chamber that are unlikely following Trabectome surgery compared with penetrating glaucoma surgery. There is limited published information on using the Trabectome for glaucoma of childhood ${ }^{24}$ although it is easier to perform than the standard: goniotomy. The latter requires vast experience in a nonpressurized anterior chamber, a proficient assistant who can counterrotate the eye and assist with visualization, and the ability to estimate the depth of the goniotomy knife's penetration into the TM. By contrast, the footplate on the Trabectome handpiece and pressurized anterior chamber would make for a more straightforward removal of TM.

\section{Exclusion Criteria}

In order to assure a stable gonioscopic view of the angle, the patient must be able to rotate their neck or entire axial core. The view must be confirmed to be clear of any corneal opacity. As noted above, active neovascular, active uveitic, or glaucoma due to elevated episcleral pressure are thought to have poor outcomes with TM ablation. Since late recurrent hyphemas have been reported, ${ }^{25}$ individuals who engage in frequent activities that increase episcleral venous pressure such as playing wind instruments or upside-down positioning have to be cautioned.

\section{Review of Technique}

Practicing visualization of the TM is a crucial step in preparing for initial Trabectome surgeries (see Figure 3). In fact, a model has been published that allows for gonioscopic visualization of donor eyes. ${ }^{26}$ Reviews are available on the topic. ${ }^{27}$

\section{Table 1: Preparation for Beginning Trabectome Surgeons}

\section{Positioning the Patient's Head}

1. Before surgery, bring the headrest close enough to the microscope to allow the telescopic arm of the microscope to swing slightly to the side of the patient's head (needed to accommodate the tilted view from the side)

2. Sitting temporally (or moving your chair slightly more toward the superior end), hold the patient's head with one hand on each temple

3. Rotate their head away from you (away from the operative eye) by about $30^{\circ}$

4. Move chair back to surgical side

\section{Setting up the Microscope}

1. Position yourself temporal to the patient's head on operative side

2. Center the head of the microscope

3. Confirm tilt knob or handle is covered

4. Tilt the head of the microscope toward yourself by 30 to $40^{\circ}$

5. Release the electromagnetic brake, manually move down

6. Bring the temporal limbus into focus by moving the microscope closer to the eye

7. Bring the limbus into full focus using fine focus.

\section{Identifying Angle Structures}

1. Confirm that the correct goniolens is handed to you (handle on the left for right-handed surgeons and handle on the right for left-handed surgeons)

2. Look through the microscope: the limbus is currently in focus-this focus will be needed to insert the Trabectome tip into the anterior chamber

3. Place goniolens on the eye

4. Focus down toward angle

5. Identify iris root, ciliary band, trabecular meshwork and distinguish from pigment along Schwalbe's line (Sampaolesi line) if present

\section{Inducing Blood Reflux to Identify Schlemm's Canal}

1. Using $0.12 \mathrm{~mm}$ forceps or a similar instrument, tap lightly on the posterior lip of the main incision to allow a small amount of fluid to egress

2. Lightly place goniolens on the eye again

3. If there is not enough fluid for a good interface, place some balanced salt solution onto the cornea

4. Observe Schlemm's canal that is now outlined with refluxed blood or is beginning to reflux where the collector channels enter Schlemm's canal

A paracentesis is fashioned as for phacoemulsification and preservativefree lidocaine is injected. No viscoelastic is required. The $1.6 \mathrm{~mm}$ main wound can be made more anterior (approximately $2 \mathrm{~mm}$ anterior to the limbus) to avoid iris prolapse as well as corneal striae during ablation. The patient's head and microscope are rotated $30-40^{\circ}$ each until adequate visualization of the angle is achieved with the goniolens (see Table 1). If the TM cannot be visualized because of minimal pigmentation, then the posterior lip of the incision can be depressed to allow a small amount of fluid to egress. The reflux of blood will highlight Schlemm's canal (see Figure 4).

The TM is engaged slightly toward the left and by pointing up at a $45^{\circ}$ angle. It is important to resist pushing outward during ablation so as to not damage the collector channels. Ablation is started at $0.8 \mathrm{~mW}$ and titrated up if necessary. ${ }^{28}$ Ablation of up to $90^{\circ}$ in both directions can be performed. A viscoadaptive substance can then be injected to fill about $20 \%$ of the anterior chamber in order to leave a crescent of viscoelastic tamponading the ablation arc to minimize postoperative hyphema (see Table 2). In narrow angles with synechiae, in order to gain access to the TM for ablation, goniosynechiolysis can be performed using the smooth base plate of the handpiece. ${ }^{29}$ 


\section{Table 2: Tips for Successful Trabectome Surgery}

Visualization

- Use an excellent microscope that can be tilted well and uses light (e.g. xenon) with a superior color rendering index and allows to see the translucent meshwork easily

- Do not use no viscoelastic before Trabectome ablation

- Induce hypotony by burping the main incision-this will cause blood to reflux into Schlemm's canal and highlight the ablation target

\section{Technique}

- Through the paracentesis, pressurize the eye with $1 \%$ lidocaine

- Do not use viscoelastic

- Place your clear corneal incision more anterior than in phacoemulsification, approximately $2 \mathrm{~mm}$ anterior the limbus and parallel to the iris

- Flare the incision on the inside to extend the reach toward the left and the right and prevent corneal folds

- Float the goniolens on the cornea, do not compress to avoid striae

- Engage slightly toward the left by pointing up in a $45^{\circ}$ angle toward the direction of ablation

- Do not push outward against the outer wall during ablation pass as this would damage the collector channels

- Ablate at least 2 clock hours and up to as much as you can safely ablate (up to $180^{\circ}$ using one incision)

\section{Reducing Hyphema}

- Reduce postoperative hyphema by tamponading the ablated sited with viscoelastic immediately after Trabectome surgery and after cataract surgery

- Pressurize the eye well after the procedure

What to Avoid

- Do not push outward during ablation, stop if view of the tip is lost

- Do not confuse the ciliary body band with the trabecular meshwork if meshwork is lightly pigmented (inducing reflux as described above helps to avoid this)

- If iris processes are dragged, back off and re-engage or skip $2 \mathrm{~mm}$ of trabecular meshwork

- Focal bleeding from the operative site indicates that an arterial injury has occurred because the eye has a pressure above that of veins; this makes it even more important to identify and ablate the trabecular meshwork anterior to the bleeding structure or IOP may be high

- A cyclodialysis cleft from Trabectome surgery is different from a traumatic one and should resolve

\section{Patient Management and Postoperative Care}

Patients should be warned that blurry vision is expected and usually lasts 3 to 10 days (see Table 3). With dense postoperative inflammation or hyphema, the visual acuity on postoperative day 1 can be as low as counting fingers. Patients may also observe that their vision is worse in the morning and gets better upon rising from shifting hyphema. Unlike with traumatic hyphemas, these patients do not require patching, atropinization, or bed rest as it will resolve spontaneously. Routine postoperative drops are similar to cataract surgery and include a fluoroquinolone, prednisolone acetate, and pilocarpine 1-2 \% four times daily. The prednisolone should be tapered weekly. Pilocarpine can be tapered to three times per day after 1 month and stopped after 2 months. Glaucoma medications can be partially or completely stopped depending on the severity of the glaucoma and postoperative IOP.

Light aerobic exercise without strenuous lifting or squatting is acceptable within a few days after surgery. As discussed above, strenuous activity and inversion can trigger recurrent hyphema. New glasses should not be dispensed until after pilocarpine has been stopped.
Table 3: Patient Management and Postoperative Care

\section{Manage Expectation by Telling Your Patient that:}

- Blurry vision is expected (can last from 3 to 10 days), and it can occasionally be as severe as counting fingers; it is a result of reflux from a patent drainage system minutes to hours after surgery when relatively low intraocular pressure may exist

- Peripheral anterior synechiae can occasionally be seen

- Vision is worse in the morning and gets better after arising (this is due to redistribution of the hyphema which will layer again with gravity)

\section{Medications-Similar to Cataract Surgery + Pilocarpine}

1. Fourth-generation fluoroquinolone

2. 1-2\% pilocarpine four times per day for 1 month, then three times per day

3. Prednisolone acetate or similar steroid four times per day for 1 week, then three times per day for 1 week then twice per day for 1 week then once per day for 1 week

4. Glaucoma medications can be partially or completely stopped depending on severity of glaucoma

Hyphema

1. Hyphema can be much reduced by placing viscoelastic along the ablated nasal angle

2. Up to $20 \%$ viscoelastic can be retained at the conclusion of cataract surgery to pressurize and seal the corneal incision; this will reduce flow back from the collector system

3. Monocular patients will have better vision early on by sleeping with the head of the bed at an incline to allow the hyphema to settle and layer; it will not change the final outcome though

4. It is not necessary to discontinue anticoagulants before or after surgery because hyphema is caused by flow back from the collectors system and not by injury

5. An eye with hyphema does not require patching, atropinization, or bed rest

6. Rarely, hyphema can occur months to years later in maneuvers or positions that increase the episcleral venous pressure e.g. prolonged inversion: this hyphema does not typically require any treatment

\section{Physical Activity}

- Aerobic exercise in the form of light treadmill running and walking is acceptable within days of surgery

- No swimming, whirlpool, or hot tub use for 1 week to reduce the infection risk

- No pilates or similar exercises that include inversion

- High-resistance wind instruments, weightlifting, and breath holding (Valsalva maneuver) increase central venous pressure so are relatively contraindicated

Fitting New Glasses

- A new prescription for glasses should only be determined once pilocarpine has been discontinued for at least 3 days

- Usually, no prescription change is seen after Trabectome in pseudophakic eyes because it is astigmatically neutral

\section{Outcomes}

In most cases, the IOP will be lowered into the midteens with a pressure reduction between 30 and $40 \% .{ }^{1}$ Although it is difficult and not accurate to combine various studies with different indications and populations, an overall average of outcomes can give some idea of the expected outcome to compare with other options. Whether or not it is combined with phacoemulsification, Trabectome has been shown to frequently lower the IOP to a final average around $16 \mathrm{mmHg}$ (see Figure 2) while decreasing the number of medications by one. ${ }^{17,30-32}$

There are limited data to help determine the success rate of the procedure, with few studies analyzing this rigorously. One definition of 
success used was final IOP $\leq 21 \mathrm{mmHg}$ with a $20 \%$ decrease from baseline while avoiding reoperation. For standalone Trabectome, this occurred in approximately $70 \%{ }^{33,34}$ of cases by the end of 1 year but then in one study fell as low as $22 \%$ by year $2 .{ }^{35}$ For phacoemulsification with Trabectome, it was approximately $90 \%{ }^{15,33}$ after 1 year and was $80 \%$ at 2 years. ${ }^{36}$

Two prospective studies indicate that pigmentary glaucomas have a better response to Trabectome than does primary open-angle glaucoma. ${ }^{19,34}$ The main difference between the two groups was success rates, with the pigmentary glaucomas having a 15-20 \% higher success rate. ${ }^{19,34}$ The improved results seen may correspond to the bulk of outflow resistance being localized only in the TM in pigmentary glaucomas. ${ }^{37}$

\section{Complications}

Trabectome has a significantly better safety profile compared with trabeculectomy and tube shunts, since there is nearly no risk for hypotony, wound leak, shallow anterior chamber, and choroidals, or the long-term risk for endophthalmitis and hardware erosion. ${ }^{38}$ The most common side effect of Trabectome is transient hyphema indicating reflux from a patent drainage system. It almost always resolves on its own within days and delayed-onset hyphema is rare. ${ }^{39-41}$ Other reported side effects include peripheral anterior synechiae in up to $24 \%$ of 37 patients. ${ }^{13}$ There are also case reports of cystoid macular edema, vitreous block, cyclodialysis cleft, endophthalmitis, and suprachoroidal hemorrhage. 13,38,42 Complication rates in these articles do not exceed $1 \% .17,43$

\section{Additional Considerations}

Current annual medical spending for glaucoma accounts for about $\$ 2.9$ billion in the US. ${ }^{44}$ As reported by a cost analysis in Ontario, Canada, after 6 years Trabectome saved $c \$ 279.23$ over monodrug and $\$ 2,424.71$ per patient versus tridrug therapy. ${ }^{45}$ Trabectome was more economical than istent but less than endoscopic cyclophotocoagulation. Combination of Trabectome with tube shunt implantation to achieve lower IOPS and a blunted hypertensive phase or repeated use of Trabectome for cases after failure is another area for further research and may be able to reduce medication dependence and IOP further while minimizing complications in eyes with multiple procedures. ${ }^{12}$

\section{Conclusion}

Trabectome surgery has been shown to be an effective option for lowering the IOP with a favorable side effect profile compared with trabeculectomy or tube shunt surgery. Since the first results in 2005, the indications have since expanded with successful outcomes in most types of primary and secondary open-and narrow angle-glaucoma. Perioperative considerations are similar to phacoemulsification with a similar speed of visual recovery. The major postoperative difference is the occurrence of hyphema, which can be reduced by leaving a crescent of viscoelastic to briefly tamponade the exposed collector channels. The IOP in successful cases is frequently lowered into the midteens while decreasing the number of medications by one. Less information is available on success rates, which are likely highest for pigmentary glaucomas.
1. Kaplowitz K, Schuman JS, Loewen NA, Techniques and outcomes of minimally invasive trabecular ablation and bypass surgery, $\mathrm{Br}\lrcorner$ Ophthalmol, 2014;98:579-85.

2. Samuelson TW, Katz $\sqcup$, Wells JM, et al., US istent Study Group. Randomized evaluation of the trabecular micro-bypass stent with phacoemulsification in patients with glaucoma and cataract, Ophthalmology, 2011:118:459-67.

3. Pfeiffer N, Garcia-Feijoo J, Martinez-de-la-Casa JM, et al A Randomized trial of a schlemm's canal microstent with phacoemulsification for reducing intraocular pressure in open-angle glaucoma, Ophthalmology, 2015;122:1283-93

4. Rosenquist R, Epstein D, Melamed S,et al., Outflow resistance of enucleated human eyes at two different perfusion pressures and different extents of trabeculotomy, Curr Eye Res, 1989:8:1233-40.

5. Quigley HA, Broman AT, The number of people with glaucoma worldwide in 2010 and 2020, Br J Ophthalmol, 2006;90:262-7.

6. Tham Y-C, Li X, Wong TY, et al., Global prevalence of glaucoma and projections of glaucoma burden through 2040: a systematic review and meta-analysis, Ophthalmology, 2014;121:2081-90.

7. Gedde SI, Herndon LW, Brandt JD, et al., Postoperative complications in the Tube Versus Trabeculectomy (TVT) study during five years of follow-up, Am I Ophthalmol, 2012:153(5):804-14.e1.

8. Khaw PT, Chiang M, Shah P, et al., Enhanced trabeculectomy: the Moorfields Safer Surgery System, Dev Ophthalmol, 2012;50:1-28.

9. Jayaram H, Strouthidis NG, Kamal DS, Trabeculectomy for normal tension glaucoma: outcomes using the Moorfields Safer Surgery technique, Br J Ophthalmol, 2015 [Epub ahead of print]

10. Administration FDA. FDA 510 (k) Database Entry Neomedix NMX 1000 (Trabectome). 2012. Available at: http://www.accessdata.fda gov/scripts/cdrh/cfdocs/cfPMN/pmn.cfm?ID=K040584 (accessed June 30,2014$)$.

11. Bussel II, Kaplowitz K, Schuman JS, Loewen NA, Trabectome Study Group. Outcomes of ab interno trabeculectomy with the trabectome by degree of angle opening, Br J Ophthalmol 2015:99:914-9.

12. Bussel II, Kaplowitz K, Schuman JS, Loewen NA, Trabectome Study Group. Outcomes of ab interno trabeculectomy with the trabectome after failed trabeculectomy $\mathrm{Br} J$ Ophthalmol 2015:99:258-62

13. Minckler DS, Baerveldt G, Alfaro MR, Francis BA, Clinical results with the Trabectome for treatment of open-angle glaucoma, Ophthalmology, 2005;112:962-7.

14. Loewen R, Lagouros E, Loewen NA, Trabectome-mediated ab interno trabeculectomy in highly complex glaucomas, Invest
Ophthalmol Vis Sci, 2014;55:3176

15. Francis BA, Winarko J, Combined Trabectome and cataract surgen versus combined trabeculectomy and cataract surgery in openangle glaucoma. Clinical and Surgical Ophthalmology 2011:29:2/3.

16. Mosaed S, Dustin L, Minckler D, Comparative outcomes between newer and older surgeries for glaucoma, Trans Am Ophthalmol SOC, 2009:107:127.

17. Jea SY, Francis BA, Vakili G, et al., Ab interno trabeculectomy versus trabeculectomy for open-angle glaucoma, Ophthalmology, 2012;119:36-42.

18. Ahuja Y, Malihi M, Sit A, Delayed-onset symptomatic hyphema after ab interno trabeculotomy surgery, Am J Ophthalmol, 2012;154:476-80.

19. Jordan JF, Wecker T, van Oterendorp C, et al., Trabectome surgery for primary and secondary open angle glaucomas, Graefes Arch Clin Exp Ophthalmol, 2013:251:2753-60.

20. Jea S, Mosaed $S$, Vold $S$, Rhee D, Effect of a failed trabectome on subsequent trabeculectomy, J Glaucoma, 2012;21:71.

21. Mosaed S, Chak G, Haider A, et al., Results of Trabectome Surgery Following Failed Glaucoma Tube Shunt Implantation: Cohort Study, Medicine, 2015:94:e1045.

22. Vold SD, Dustin L, Trabectome Study Group, Impact of laser trabeculoplasty on Trabectome ${ }^{\oplus}$ outcomes, Ophthalmic Surg Lasers Imaging, 2010:41:443-51.

23. Neiweem A, Bussel II, Schuman JS, et al., Trabectome surgery calculator: additive effect from combining phacoemulsification with trabectome in a matched comparison to trabectome alone. submitted.

24. Morales J, Shahwan S Al, Odhayb S Al, et al., Current surgical options for the management of pediatric glaucoma, J Ophthalmol, 2013:2013:763735

25. Ahuja Y, Malihi M, Sit AJ, Delayed-onset symptomatic hyphema after ab interno trabeculotomy surgery, Am J Ophthalmol, 2012;154:47-480.e2

26. Patel SP, Sit AJ, A practice model for trabecular meshwork surgery, Arch Ophthalmol, 2009;127:311-3.

27. Shareef S, Alward W, Crandall A, et al., Intra-operative gonioscopy: a key to successful angle surgery, Expert Rev Ophthalmol, 2014;9:515-27.

28. Francis $B$, See $R$, Rao N, et al., Ab Interno Trabeculectomy: Development of a novel device (Trabectome (TM)) and surgery for open-angle glaucoma, J Glaucoma, 2006;15:68-73.

29. Kaplowitz K, Chen X, Loewen N, Two year results for 180 degree trabectome ablation. Poster \#24. In: Vol American Glaucoma Society Annual Meeting, 2013.

30. Minckler D, Mosaed S, Dustin L, Francis B, Trabectome (trabeculectomy —internal approach): Additional experience and extended follow-up, Trans Am Ophthalmol Soc, 2008;106:149.

31. Ting JLM, Damji KF, Stiles MC, Trabectome Study Group, Ab interno trabeculectomy: outcomes in exfoliation versus primary openangle glaucoma, J Cataract Refract Surg, 2012;38:315-23.

32. Werth JP, Gesser $\mathrm{C}$, Klemm M, Diverse effectiveness of the trabectome for different types of glaucoma, Klin Monbl Augenheilkd, 2015;232:72-8.

33. Mosaed S, Rhee D, Filippopoulos T, Trabectome outcomes in adult open-angle glaucoma patients: one-year follow-up, Clin Surg Ophthalmol, 2010;28:5-9.

34. Ting J, Damij K, Stiles M, Ab interno trabeculectomy: Outcomes in exfoliation versus primary open-angle glaucoma, J Cataract Refract Surg, 2012;38:315-23.

35. Jea SY, Francis BA, Vakili G, et al., Ab interno trabeculectomy versus trabeculectomy for open-angle glaucoma, Ophthalmology, 2012:119:36-42

36. Francis BA, Trabectome combined with phacoemulsification versus phacoemulsification alone: a prospective, non-randomized controlled surgical trial, Clin Surg Ophthalmol, 2010;28:1-7.

37. Speakman JS, Pigmentary dispersion, Br J Ophthalmol, 1981:65:249-51.

38. Jea SY, Francis BA, Vakili G, et al, Ab interno trabeculectomy versus trabeculectomy for open-angle glaucoma, Ophthalmology, 2012:119:36-42

39. Ahuja Y, Malihi M, Sit AJ, Delayed-onset symptomatic hyphema after ab interno trabeculotomy surgery. Reply, Am J Ophthalmol, 2013;155:779.

40. Kassam F, Stechschulte AC, Stiles MC, et al., Delayed spontaneous hyphemas after ab interno trabeculectomy surgery for glaucoma, I Glaucoma, 2014:23:660-1.

41. Parekh AS, Weinreb RN, Dorairaj SK, Delayed-onset symptomatic hyphema after ab interno trabeculotomy surgery, Am J Ophthalmol, 2013;155:778-9.

42. Jordan JF, Wecker T, van Oterendorp C, et al., Trabectome surgery for primary and secondary open angle glaucomas, Graefes Arch Clin Exp Ophthalmol, 2013;251:2753-60.

43. Vold SD, Ab interno trabeculotomy with the trabectome system: what does the data tell us? Int Ophthalmol Clin, 2011:51:65-81.

44. Rein DB, Zhang P, Wirth KE, et al., The economic burden of major adult visual disorders in the United States, Arch Ophthalmol, 2006;124:1754-60

45. Iordanous Y, Kent JS, Hutnik CML, Malvankar-Mehta MS, Projected cost comparison of Trabectome, iStent, and endoscopic cyclophotocoagulation versus glaucoma medication in the Ontario Health Insurance Plan, J Glaucoma, 2014:23:0112-e118. 